\title{
The Clinical Management of Complex Pseudoaneurysms of the Femoral Artery
}

\author{
Zaiwei Zhang ${ }^{1,2}$, Hongyan Tian ${ }^{1, *}$, Qiang Ma ${ }^{1}$, Junbo Zhang ${ }^{1}$ and Hua Tian ${ }^{1}$ \\ ${ }^{1}$ Department of Cardiovascular Medicine, First Affiliated Hospital, Medical school of Xi'an Jiaotong University, Xi'an 710061, China \\ ${ }^{2}$ Cadre Health Care Department of Mianyang Central Hospital, Mianyang, 621000, China
}

"Corresponding author: Hongyan Tian, Department of Cardiovascular Medicine, First Affiliated Hospital, Medical school of Xi'an Jiaotong University, Xi'an 710061, China, Tel: 0086-29-85324028; Fax: 0086-29-85323234; E-mail: zhzw2001@stu.xjtu.edu.cn

Received date: July 16, 2014, Accepted date: Aug 14, 2014, Published date: Aug 21, 2014

Copyright: @ 2014 Zhang Z et al. This is an open-access article distributed under the terms of the Creative Commons Attribution License, which permits unrestricted use, distribution, and reproduction in any medium, provided the original author and source are credited.

\begin{abstract}
Objective: To evaluate the clinical course of patients treated for multiple perforations, complex femoral artery pseudoaneurysms.

Methods: Seven patients with multiple perforations, complex pseudoaneurysms after femoral artery access for interventional procedures were treated. Ultrasound-guided thrombin injection (UGTI) was first used for treatment. Patients failing repeated UGTIs were treated with fibrin sealant injections.

Results: Five of the seven patients treated with UGTIs had rapid thrombus formation within the pseudoaneurysm. Two patients refractory to UGTI had successful treatment using fibrin sealant injection. There were no complications and the overall cure rate was $100 \%$.

Conclusions: Treatment of complex femoral artery pseudoaneurysms is safe and effective. UGTI and fibrin sealant are useful in the treatment of complex pseudoaneurysms.
\end{abstract}

Keywords: Pseudoaneurysm; Femoral artery; Sequential therapy

\section{Introduction}

As the use of radiologic interventions increase, so does the occurrence of iatrogenic pseudoaneurysms. The occurrence rate of pseudoaneurysms after vascular procedures has been reported as $0.6 \%$ - $6.0 \%$ [1]. If a pseudoaneurysm is not treated properly, it can cause severe complications, including rupture, distal embolization, local pain, neuropathy and local skin ischemia. Simple pseudoaneurysms are treated with local compression or ultrasound-guided thrombin injections (UGTIs) [2]. The management of complex pseudoaneurysms, which have multiple perforation and large cavity, is not clear. Herein we summarize our experience with treating complex pseudoaneurysms by describing a safe and effective treatment approach.

\section{Materials and Methods}

\section{General Information}

This study was approved by hospital ethics committee and informed consent was obtained in all patients. Seven patients with complex pseudoaneurysms after percutaneous coronary interventions were admitted for treatment. (The total number of lumen was 38 . Five cases had 2 perforations and 3-5 cysts. One case had 3 perforations and 8 cysts. One case had 4 breaks and 10 cysts.) There were 3 male patients and 4 female patients with a mean age of 58.5 years (range: 45-78 years). The pseudoaneurysms were 2-10 days old (median duration, 3.9 days). The largest pseudoaneurysm was $12.0 \mathrm{~cm} \times 5.8$ $\mathrm{cm} \times 5.2 \mathrm{~cm}$ and the smallest was $2.2 \mathrm{~cm} \times 1.4 \mathrm{~cm} \times 1.1 \mathrm{~cm}$. The pseudoaneurysm diameters ranged from 1.5 to $5.0 \mathrm{~mm}$. Color Doppler
Flow Imaging (CDFI) demonstrated flow entering the pseudoaneurysm from the femoral artery. Bidirectional flow was seen in the opening of the pseudoaneurysms (Figure 1).

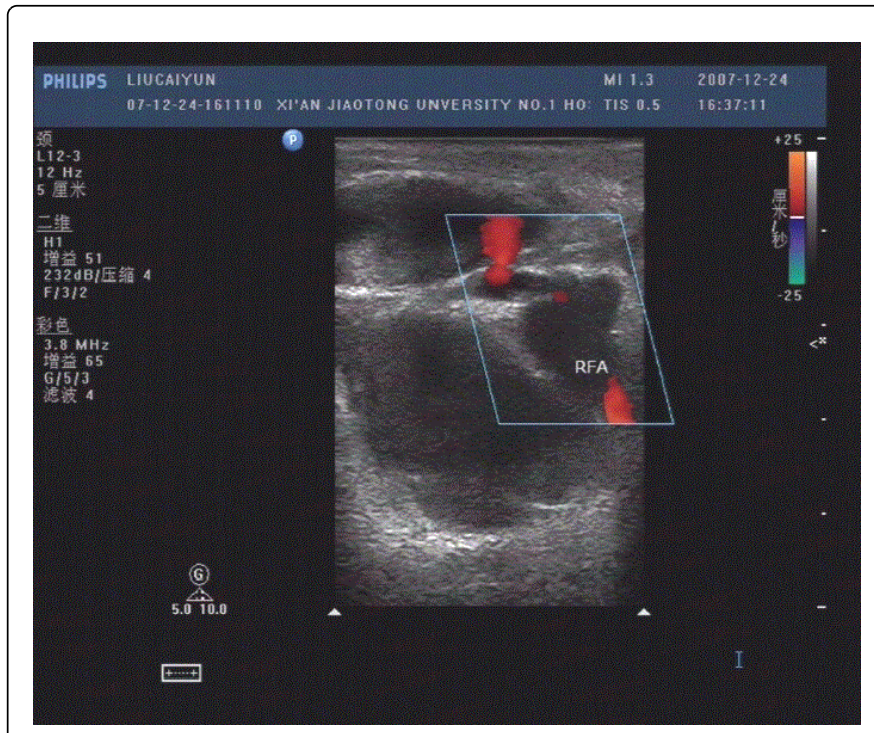

Figure 1: Color flow seen through the femoral artery rupture into the pseudoaneurysm on ultrasound.

The pseudoaneurysm in 1 patient was not imaged well due to scar formation in the right inguinal region and the large size of the pseudoaneurysm. Therefore digital subtraction angiography (DSA) was used to evaluate the pseudoaneurysm. Two-dimensional DSA 
Citation: Zhang Z, Tian H, Ma Q, Zhang J, Tian H (2014) The Clinical Management of Complex Pseudoaneurysms of the Femoral Artery. J

Page 2 of 3

demonstrated flow from the femoral artery suggesting the pseudoaneurysm had formed from the right femoral artery (Figure 2).

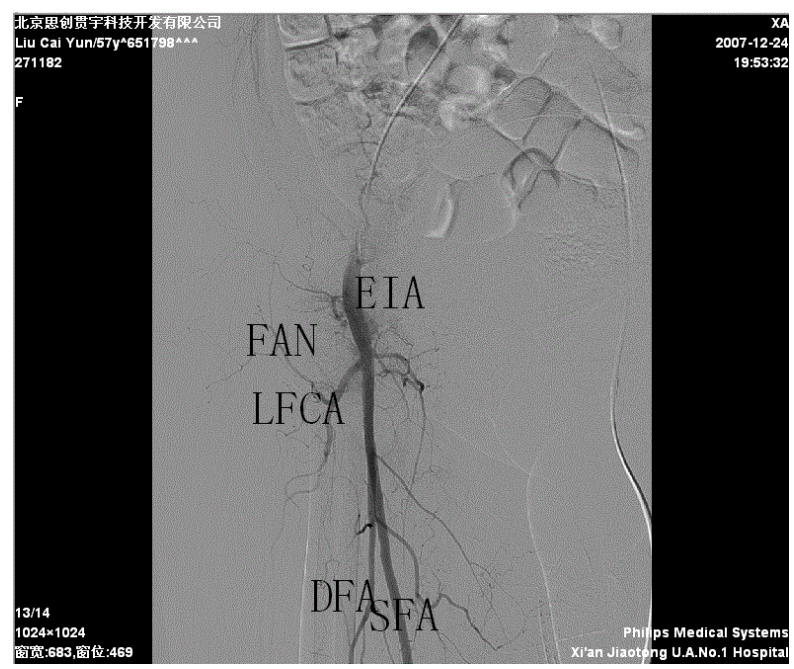

Figure 2: Extravasation of contrast around the femoral artery pseudoaneurysm on DSA. DFA, deep femoral artery; EIA, external iliac artery; SFA, superficial femoral artery; FAN, Pseudoaneurysm; LFCA, lateral circumflex femoral artery; Arrow indicates the pseudoaneurysm.

Three-dimensional DSA demonstrated multiple perforations (Figure 3). The clinical manifestation was discoloration on the surface of punctured skin, obvious tenderness and pulsatile masses with increasing size, some of which showed tremor and murmur.

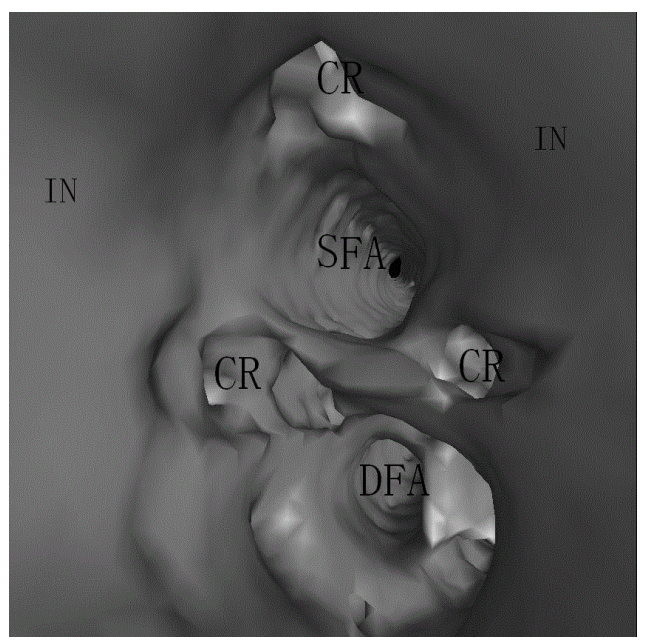

Figure 3: Three-dimensional DSA demonstrating multiple breaks of pseudoaneurysms. CR, Break of pseudoaneurysm; DFA, deep femoral artery; SFA, superficial femoral artery; IN, lining of the blood Vessels

\section{Methods and Procedures}

A GE vivid7 color Doppler ultrasound with a probe frequency of 3-9 $\mathrm{MHz}$ was used for imaging. Thrombin was injected into the pseudoaneurysm cavity under ultrasound guidance. Thrombin with a concentration of 100-200 U/ml was used for treatment. Fibrin sealant was injected into the pseudoaneurysm when thrombin injection failed.

Patients were positioned in the supine position, with limbs straightened. Femoral artery lesions were imaged using real-time twodimensional and color Doppler ultrasound. The position, number, size, blood supply, perforated artery, and body of aneurysms were evaluated. The path of the treatment needle was chosen according to the relationship of the pseudo aneurysm to the femoral artery, using color Doppler ultrasound. T-branched tubing was attached to the 20G puncture needle, allowing injection of normal saline and or thrombin. Normal saline was injected when the tip of the needle was observed to be in the cavity of the pseudo aneurysm. Thrombin was slowly injected until the perforation was closed, and the needle then removed. Color Doppler ultrasound was used to ensure there was no blood flow into the pseudoaneurysm after treatment. High intensity echoes were seen filling the pseudoaneurysm after successful treatment (Figure 4).

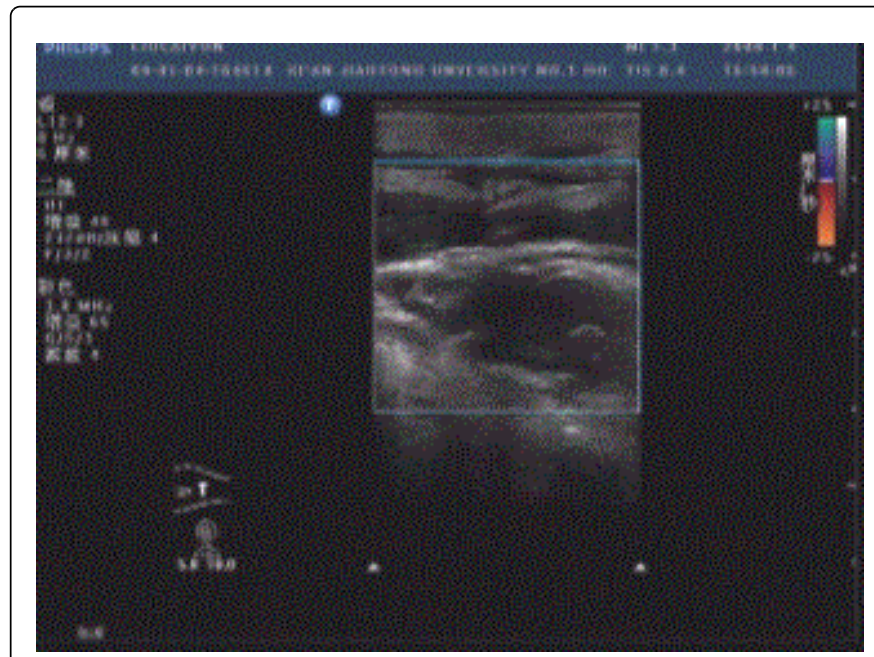

Figure 4: Ultrasound showed the femoral artery rupture disappeared, no color blood flow through the femoral artery rupture into the pseudoaneurysm.

Pressure was applied for 2 to $5 \mathrm{~min}$, using the ultrasound transducer, if residual flow was still present. The treated pseudoaneurysm was examined until no flow was seen. The process was repeated as necessary. Thrombin treatment failures were treated with fibrin sealant in a similar fashion.

\section{Results}

Seven patients underwent injection of thrombin into the pseudoaneurysm cavity. Closure of the pseudoaneurysm occurred after 1 injection in 5 patients. Two patients failed thrombin treatment. Both of these patients were treated with 1 injection of fibrin sealant into the pseudoaneurysm cavity. Closure of the pseudoaneurysm occurred in one of the two patients. The remaining patient was treated successfully using a second injection of fibrin sealant into the pseudoaneurysm under DSA guidance. The overall success rate was 
$100 \%$. The two patients who failed thrombin treatmentwere one patient with multiple interventional therapies and the other patient of inguinal region after surgical operation, local anatomy was not clear.

Patients treated with thrombin received a mean of $580 \mathrm{U}$ (range: 300-1000 U). The two patients receiving fibrin sealant received $1000 \mathrm{U}$ fibrin sealant each. All patients tolerated the procedures well. All patients had normal femoral artery and arteria dorsalis pedis pulses after their procedures.

\section{Discussion}

In this study we reported the successful treatment of complex pseudoaneurysms of the femoral artery. We found that in the majority of the cases (5 of 7) thrombin injection was successful to seal these lesions, but for the two patients who failed, those patients were successfully salvaged with fibrin injection. The dose of thrombin required was higher than that used in simple lesions. Patients were followed closely for related complications, such as distal artery thrombosis, but none was seen.

Percutaneous femoral artery access is widely used in the diagnosis and treatment of cardiovascular disease. Use of this method can lead to blood leakage from the puncture site, forming one or more cavities or pseudoaneurysms. Pseudoaneurysms do not resolve spontaneously. Common complications include compression of adjacent structures, rupture, embolism, and skin necrosis. The treatment of pseudoaneurysms includes local compression and UGTI.

Thrombin injection into a pseudoaneurysm cavity is widely used. The success rate is $74 \%$ to $86 \%$ with common pseudoaneurysms. The success rate in patients with pseudoaneurysms larger than $2 \mathrm{~cm}$ in diameter, obese patients, and patients taking anticoagulant therapy is $25 \%$ to $35 \%$. The recurrence rate is $20 \%$ in these patients [3-5].
Coincidentally we found thrombin injection also failed in 2 patients we treated. We found that fibrin sealant injection was able to close these leaks. These patients had complex pseudoaneurysms which did decrease in size after treatment with thrombin. Imaging after repeat injections with thrombin demonstrated no thrombus in the pseudoaneurysm cavity, presumably due to consumption of fibrinogen. Fibrin sealant contains both fibrinogen and thrombin, and is more useful in patients with a fibrinogen consuming condition. Fibrin sealant worked well in both patients we treated. We believe our small case series demonstrates a novel sequential approach in the initial treatment of complex pseudoaneurysm using UGTI and fibrin injection in refractory cases.

\section{References}

1. Görge G, Kunz T, Kirstein M (2003) A prospective study on ultrasoundguided compression therapy or thrombin injection for treatment of iatrogenic false aneurysms in patients receiving full-dose anti-platelet therapy. Z Kardiol 92: 564-570.

2. He GB, Liu LW, Qi HY, Zhang MM, Wang L (2008) A Comparative Study of Ultrasound-guided Compression Repair and Ultrasound-guided Thrombin Injection in the Treatment of Pseudoaneurysms. Chin J Clin Pract Med 12: 27-29.

3. Coley BD, Roberts AC, Fellmeth BD, Valji K, Bookstein JJ, et al. (1995) Postangiographic femoral artery pseudoaneurysms: further experience with US-guided compression repair. Radiology 194: 307-311.

4. Eisenberg L, Paulson EK, Kliewer MA, Hudson MP, DeLong DM, et al. (1999) Sonographically guided compression repair of pseudoaneurysms: further experience from a single institution. AJR Am J Roentgenol 173: 1567-1573.

5. O'Sullivan GJ, Ray SA, Lewis JS, Lopez AJ, Powell BW, et al. (1999) A review of alternative approaches in the management of iatrogenic femoral pseudoaneurysms. Ann R Coll Surg Engl 81: 226-234. 